\title{
Focal or Macro-reentrant (Dual-loop) Atrial Tachycardia? The Role of the Ligament of Marshall
}

\author{
FRANCESCO PERUZZA, MD, ${ }^{1}$ CARLO ANGHEBEN, MD, ${ }^{1}$ MASSIMILIANO MAINES, MD, ${ }^{1}$ \\ PAOLO MOGGIO, MD, ${ }^{1}$ DOMENICO CATANZARITI, MD, ${ }^{1}$ CAMILLA BONVICINI, ${ }^{2}$ STEFANO INDIANI, ${ }^{2}$ \\ and MAURIZIO DEL GRECO, MD, FHRS ${ }^{1}$
}

'Santa Maria del Carmine Hospital, Rovereto, TN, Italy

${ }^{2}$ Abbott, Milan, Italy

KEYWORDS. Arrhythmogenic right ventricular cardiomyopathy, atrial tachycardia, left atrial

ISSN 2156-3977 (print) ISSN 2156-3993 (online) CC BY 4.0 license

(C) 2021 Innovations in Cardiac Rhythm Management
A 56-year-old man was referred to our institution for cardiac ablation because of several episodes of palpitations and thoracic pain with an electrocardiogram suggestive for left atrial tachycardia (160 bpm). He was affected by arrhythmogenic right ventricular cardiomyopathy (ARVC) with left ventricular involvement and a plakophillin-2 gene mutation. A cardiac magnetic resonance imaging scan showed biventricular fibro-fatty infiltration with matching late gadolinium enhancement.

An electrophysiological study was performed in combination with electroanatomic mapping (EnSite Precision $^{\mathrm{TM}}$ ). An activation map of the left atrium during tachycardia was created with the Advisor ${ }^{\mathrm{TM}}$ HD Grid

Ms. Bonvicini and Mr. Indiani are employees of Abbott. The other authors report no conflicts of interest for the published content.

Address correspondence to: Francesco Peruzza, MD.

Email: francesco.peruzza@apss.tn.it.
Mapping Catheter, Sensor Enabled ${ }^{\mathrm{TM}}$. The region of earliest activation was identified at the base of the left atrial appendage (LAA), confirmed also by the EnSite ${ }^{\mathrm{TM}}$ LiveView Dynamic Display. The activation wavefront was suggestive for a focal-origin tachycardia from the LAA with an uncommon line of block between the LAA and left superior pulmonary vein along the left atrial roof (Figure 1). However, we could not exclude a macroreentrant (dual-loop) tachycardia (Video 1) with a slowconduction isthmus inside the vein of Marshall with its fibrous and muscular component (Marshall bundle) as supported by the presence of fragmented potential along the line of block.

Ablation with $25 \mathrm{~W}$ (TactiCath ${ }^{\mathrm{TM}}$ Contact Force Ablation Catheter, Sensor Enabled ${ }^{\mathrm{TM}}$ ) at the earliest activation site terminated the tachycardia within three seconds. At six months of follow-up, no recurrence of the arrhythmia was observed. 


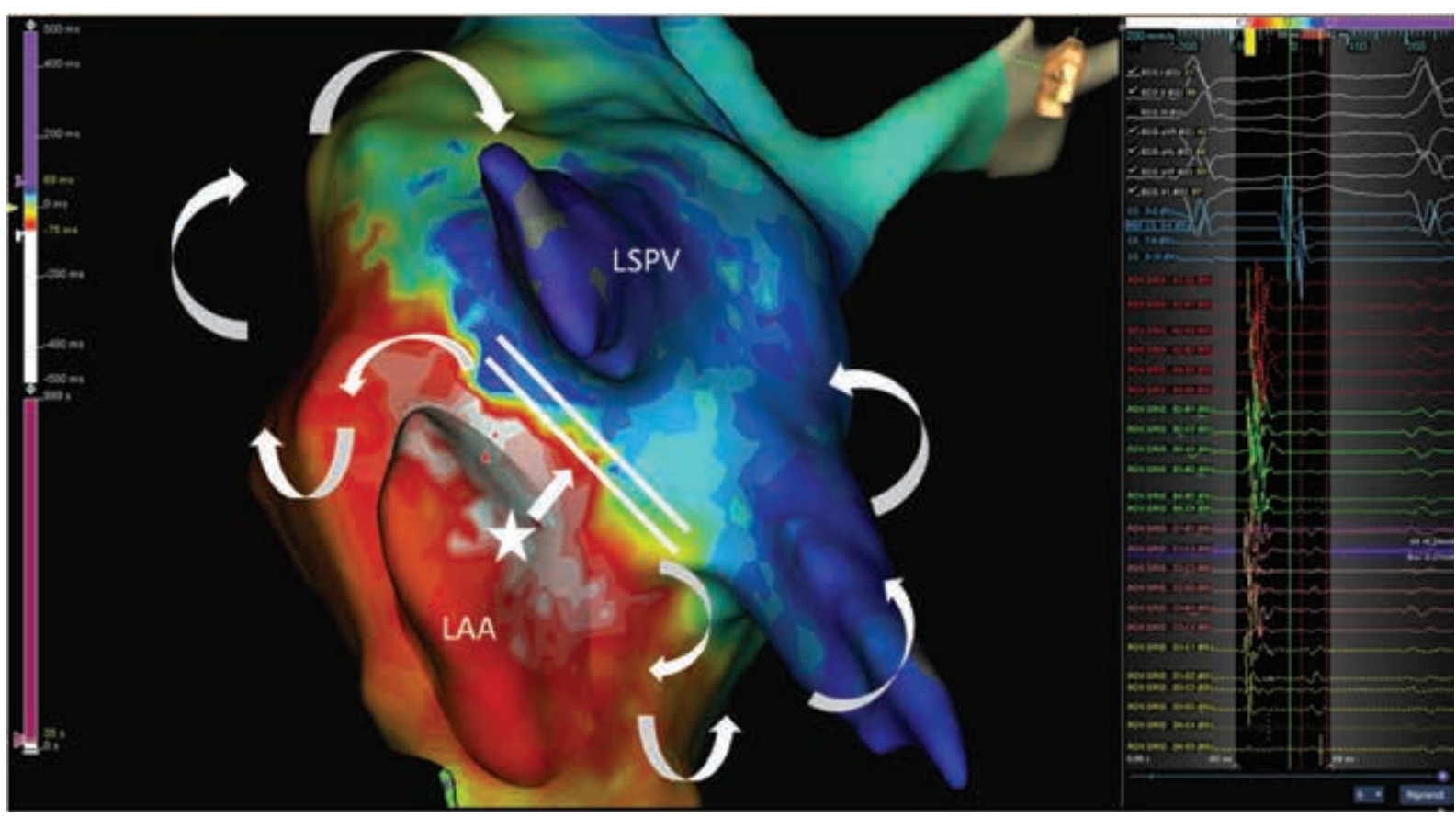

Figure 1: Left atrium activation mapping with the AdvisorTM HD Grid Catheter. The activation wavefront was suggestive for a focal origin of tachycardia from the LAA with an uncommon line of block between the LAA and left superior pulmonary vein along the left atrial roof. LAA: left atrial appendage; LSPV: left superior pulmonary vein. 